\title{
Measurement of a Second Harmonic Distortion of the Detected Signal in an IM-DD Fiber Optic Link when the input IM is second harmonic contaminated
}

\author{
Chiranjib Ghosh and Taraprasad Chattopadhyay \\ Department of Physics, Visva-Bharati University, Santiniketan -731235, Birbhum, West Bengal, India
}

Received July 17, 2015; accepted August 13, 2015; published September 30, 2015

\begin{abstract}
In this letter, we have measured a second harmonic distortion of the detected optical signal in an IM-DD fiber optic link when the input intensity modulation RF signal contains a small amount of second harmonic distortion. The plot of output vs. input second harmonic distortion is linear. The output second harmonic distortion is smaller than the input second harmonic distortion. This is due to the existence of a small negative slope in the modulation response of the FP laser diode over the $100 \mathrm{MHz} \div 200 \mathrm{MHz}$ frequency band.
\end{abstract}

With the rapid development of an optical communication system in the $21^{\text {st }}$ century, the research on high speed optical modulation and detection is being carried out all over the globe. The simplest, widely used and commercially viable fiber optic communication is the IM-DD communication. The modulation format used here is the intensity modulation (IM) and the detection scheme employed is the direct detection (DD) in a photodiode. In any communication system, message signal distortion at the output is an important parameter for assessing the reliability of the communication link.

Survey of relevant literature indicates that message signal distortion related studies have been done by some authors [1-5]. Investigation of harmonic distortion, intermodulation distortion, signal clipping and effect of RIN on subcarrier multiplexed IM-DD optical link has been carried out by some authors [1-3]. Harmonic distortion and intermodulation distortion in direct modulation arises from the nonlinearity of light power vs. Laser diode (LD) bias current characteristics (L-I curve). No investigation seems to be carried out when the input RF IM signal is itself second harmonic contaminated.

The experimental set up using a laser test bench supplied by Thorlabs is shown in Fig. 1 (optical power meter: PM100D, pigtailed LD mount: LM9LP, PD detector: DEC01CFC).

The Fabry-Perot laser diode (FPLD) lasing at the central wavelength of $1557.1 \mathrm{~nm}$ operates at $23^{\circ} \mathrm{C}$ which is maintained by a TEC controller (ITC4001). The DC bias current $\left(I_{d c}\right)$ and the RF modulation current $\left(I_{m o d}\right)$ are applied to the LD through a bias tee. The LD output is intensity modulated (IM). The intensity modulated lightwave is fed to an InGaAs photodiode (PD) having a bandwidth of $1.2 \mathrm{GHz}$. The PD output current is proportional to the input optical power. The output of the PD is connected to a load resistance $R_{L}$. The IM lightwave is detected by a PD and its power spectrum is displayed in an Agilent microwave spectrum analyzer (E4403B, 9kHz-3 GHz). Input and output second harmonic distortion can be measured using this spectrum analyzer. The fundamental modulation frequency is $100 \mathrm{MHz}$ and the second harmonic is at $200 \mathrm{MHz}$.

The $1557.1 \mathrm{~nm}$ wavelength falls in the C-band $(1525 \mathrm{~nm} \div 1565 \mathrm{~nm})$ of the IR spectrum. Fiber loss is minimal at this wavelength. Also, optical amplifiers like EDFA are available at this wavelength and DWDM communication can be implemented over this band. Since $1557.1 \mathrm{~nm}$ wavelength falls in the telecommunication band, message signal distortion is of serious concern. As such, second harmonic distortion being a major source of harmonic distortion in IM-DD fiber-optic link has motivated us to study its effect on the link. We have used a Fabry-Perot laser diode since it is cheap.

The $1557.1 \mathrm{~nm}$ wavelength has also application in infrared radio-astronomy. At this wavelength, the atmospheric opacity is small, sky transparency is high and sky brightness is low so that ground based astronomical observations can be made.

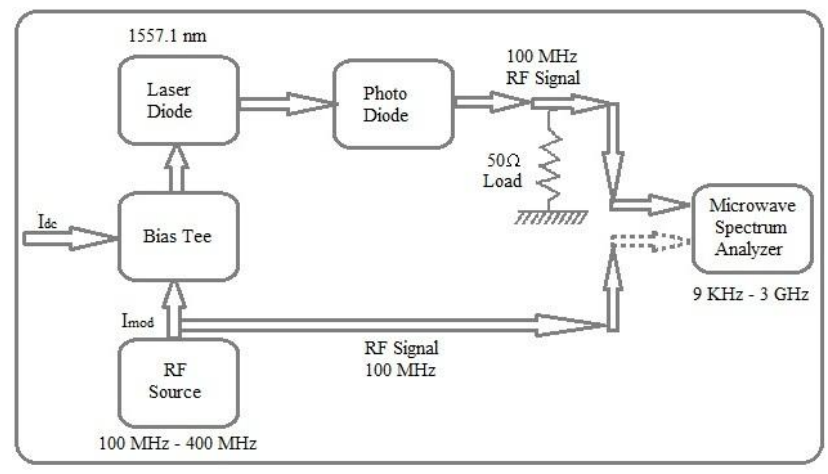

Fig. 1. Experimental setup for measuring second harmonic distortion in an IM-DD Fiber-Optic link. 
In this letter, we have measured the detected RF signal distortion in an IM-DD fiber optic link when the input message signal contains a small amount of second harmonic distortion. The dependence of output distortion on input message signal distortion has been found to be linear in the logarithmic scale.

Let the RF modulating signal voltage be represented as

$V_{m o d}=V_{m 1} \sin \omega_{m} t+V_{m 2} \sin 2 \omega_{m} t$,

where $V_{m}$ is the voltage amplitude and $\omega_{m}$ is the radian frequency of the fundamental RF signal. The powers of the RF signal and the second harmonic are

$P_{\text {mod } f}=\frac{V_{m l^{2}}}{2 R}$

and

$$
P_{\bmod 2}=\frac{V_{m 2}^{2}}{2 R},
$$

respectively.

Here $R$ is the input impedance of the LD at its direct modulation port. For the LD under consideration, different physical parameters are shown in Table 1.

Table 1: Values of different physical parameters

\begin{tabular}{|l|c|}
\hline Lasing wavelength $\left(\lambda_{\mathrm{c}}\right)$ & $1557.1 \mathrm{~nm}$ \\
\hline LD temperature & $23^{\circ} \mathrm{C}$ \\
\hline Threshold current of LD & $41.6 \mathrm{~mA}$ \\
\hline DC bias current of LD & $50 \mathrm{~mA} \div 100 \mathrm{~mA}$ \\
\hline InGaAs PD bandwidth & $1.2 \mathrm{GHz}$ \\
\hline LD modulation input resistance & $50 \Omega$ \\
\hline Load resistance of PD & $50 \Omega$ \\
\hline Responsivity of photodiode & $0.88 \mathrm{~mA} / \mathrm{mW}$ \\
\hline
\end{tabular}

$P_{\text {mod }}$, measured by the MSA, contains fundamental frequency at $100 \mathrm{MHz}$ and a second harmonic at $200 \mathrm{MHz}$. The intensity or power modulation of LD is expressed as

$$
P(t)=P_{0}\left(1+m_{1} \sin \omega_{m} t+k m_{2} \sin 2 \omega_{m} t\right),
$$

where $m_{1}$ and $m_{2}$ are the fundamental and second harmonic intensity or power modulation indices and $k$ is a slope parameter of the LD modulator for the second harmonic. The detected signal voltage is calculated as

$V_{D 0}=\eta P(t) R_{L}=\eta R_{L} P_{0}\left(1+m_{1} \sin \omega_{m} t+k m_{2} \sin 2 \omega_{m} t\right)$,

where $\eta$ is the responsivity of the PD, $R_{L}$ is the load resistance of the PD. The factory supplied data on the LD modulation response shows that there exists a very small downward slope in the modulation response over the modulation frequency band $100 \mathrm{MHz} \div 200 \mathrm{MHz}$.This is taken into account by the slope parameter $k$. The detected fundamental and second harmonic voltages of the output of the PD are given as
$V_{D 1}=\eta R_{L} P_{0} m_{1}$

and

$V_{D 2}=\eta \mathrm{R}_{\mathrm{L}} P_{0} k m_{2}$

respectively.

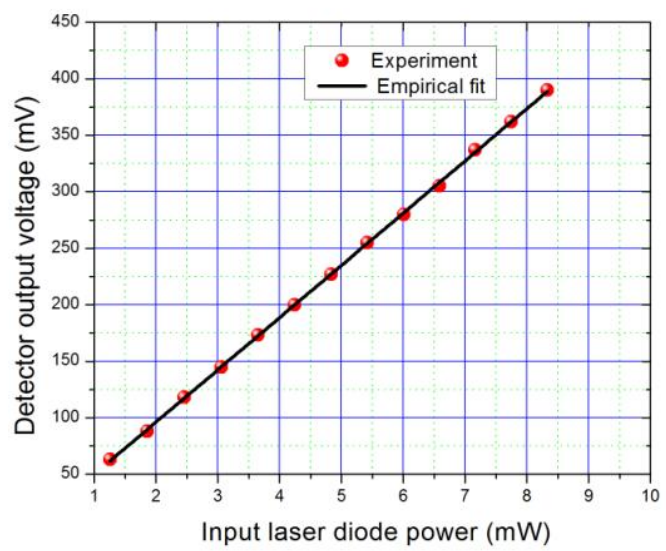

Fig. 2. InGaAs photodiode response.

To calculate $\eta$, we have measured the detected output voltage in response to input optical power, which is plotted in Fig. 2. The slope of this linear plot is proportional to $\eta$.

Now the second harmonic distortion of the input RF signal $\left(D_{\text {in }}\right)$ and the same for the detected RF signal output $\left(D_{\text {out }}\right)$ are given in $\mathrm{dB}$ by

$D_{\text {in }}=20 \log _{10}\left(\frac{V_{m 2}}{V_{m 1}}\right)$,
$D_{\text {out }}=20 \log _{10}\left(\frac{k m_{2}}{m_{1}}\right)$.

Now, we can write

$\frac{V_{m 2}}{V_{m 1}}=\left(P_{m 2} / P_{m 1}\right)^{1 / 2}$

Then, from Eq. (8) and Eq. (10) we write

$$
\begin{aligned}
D_{i n} & =10 \log _{10}\left(P_{m 2} / P_{m 1}\right) \mathrm{dB}= \\
& =10 \cdot\left(\log _{10} P_{m 2}-\log _{10} P_{m 1}\right) \mathrm{dB}= \\
& =\left(\left.P_{m 2}\right|_{\mathrm{dBm}}-\left.P_{m 1}\right|_{\mathrm{dBm}}\right) \mathrm{dB} .
\end{aligned}
$$

$P_{m 1}$ and $P_{m 2}$ can be given in $\mathrm{dBm}$ taking the reference power as $1 \mathrm{~mW}$.

Similarly,

$$
\begin{aligned}
D_{\text {out }} & =20 \log _{10}\left(V_{D 2} / V_{D 1}\right)=20 \log _{10}\left(P_{d 2} / P_{d 1}\right)^{1 / 2}= \\
& =10\left(\log _{10} P_{d 2}-\log _{10} P_{d 1}\right) \mathrm{dB}= \\
& =\left(\left.P_{d 2}\right|_{\mathrm{dBm}}-\left.P_{d 1}\right|_{\mathrm{dBm}}\right) \mathrm{dB} .
\end{aligned}
$$




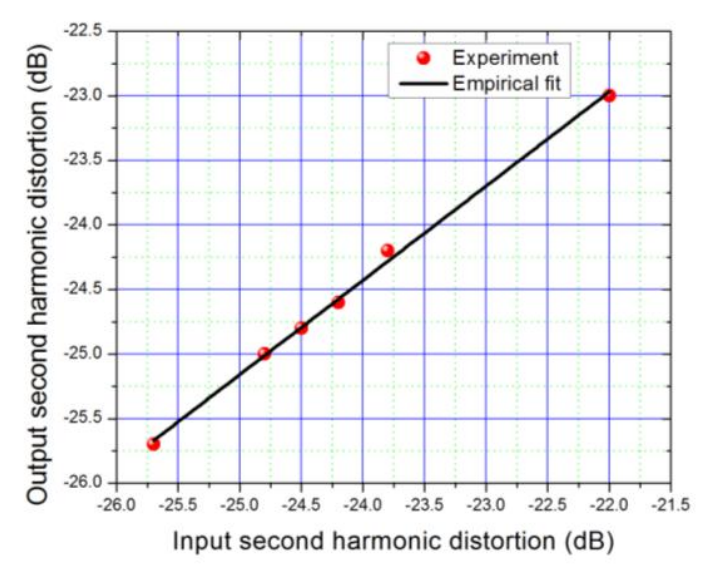

Fig. 3. Output second harmonic distortion as a function of input second harmonic distortion in an IM-DD fiber optic link.

The measured values of output second harmonic distortion, $D_{\text {out }}$, are plotted as a function of measured values of input second harmonic distortion, $D_{i n}$, in Fig. 3. The plot in units of $\mathrm{dB}$ is linear. The plot has a positive slope. The output second harmonic distortion is smaller than the input second harmonic distortion because of the negative slope factor $k$ in the modulation response over the frequency band of $100 \mathrm{MHz} \div 200 \mathrm{MHz}$. The value of $k$ has been determined experimentally from the plot. The average value of $k$ comes out as $k=-0.3 \mathrm{~dB}$.

The fundamental modulation index is calculated as

$m_{i}=I_{\text {mod }} /\left(I_{\text {bias }}-I_{\text {th }}\right)$,

where $I_{\text {mod }}=V_{\text {mod }} / R$. Here, $I_{\text {mod }}$ is fundamental modulation current, $V_{\text {mod }}$ is fundamental modulation voltage, $I_{\text {bias }}$ is LD bias current and $I_{t h}$ is LD threshold current.

Apparently, it seems that the second harmonic problem can be removed through filtering and external modulation. But, external modulator nonlinearity gives rise to harmonic distortion of the modulated signal unless the driving signal amplitude is kept at a very low level, which leads to a low modulation index. For a monochromatic RF source, the second harmonic component can be eliminated through selective filtering. However, for a multifrequency source, many such filters are required, leading to design complexity.

Higher order harmonic distortions are smaller than the second harmonic distortion. The second harmonic distortion, as observed in our experiment, is the major source of distortion. However, third and higher order distortions need further investigation.

The input RF source is inherently contaminated by a second harmonic. When the fundamental modulation frequency is chosen as $100 \mathrm{MHz}$, the second harmonic at
$200 \mathrm{MHz}$ is automatically generated in the synthesized source in a small amount which causes second harmonic distortion.

In this letter, we have measured the second harmonic distortion of the detected signal in an IM-DD fiber optic link when the input modulation contains a small amount of second harmonic distortion. The variation of output second harmonic distortion with the input second harmonic distortion turns out to be of linear nature. The measured second harmonic distortion is slightly smaller than the input second harmonic distortion. This is due to the existence of a small negative slope factor in the modulation response of the laser diode over the modulation frequency band of $100 \mathrm{MHz}$ to $200 \mathrm{MHz}$.

From our experiment it is inferred that the input second harmonic distortion level does not change much during its transmission over the IM-DD link. For a single tone modulation, an optical notch filter can be used before detection to eliminate second harmonic distortion and for multitone modulation - an optical band stop filter.

The authors acknowledge the financial assistance received from the Council of Scientific and Industrial Research, New Delhi, India, under the project 03(1291)/13/EMR-II.

\section{References}

[1] G. Qazi, A.K. Sharma, H.N. Shah, M.-u.-din, Optik 125, 2148 (2014).

[2] G. Qazi, A.K. Sharma, H.N. Shah, M.-u.-din, Optik 125, 1629 (2014).

[3] G. Qazi, A.K. Sharma, H.N. Shah, M.-u.-din, Optics Comm. 319, 178 (2014).

[4] V. Sharma, Optik 121, 1545 (2010).

[5] J.H. Schaffner,W.B. Bridges, J. Lightwave Techn. 11, 3 (1993).

[6] L. Roselli,V. Borgioni, F. Zepparelli, M. Ambrosi, M. Comez, P. Faccin, A. Casini, J. Lightwave Techn. 21, 1211 (2003).

[7] A.J. Rainal, J. Lightwave Techn. 14, 474 (1996). 